\title{
Docetaxel for the post-surgery treatment of patients with node-positive breast cancer
}

\author{
Shubham Pant ${ }^{\prime}$ \\ Meena P Chilukuri² \\ Bhuvaneswari Ramaswamy' \\ 'Arthur G. James Cancer Hospital \\ and Richard J. Solove Research \\ Institute, Ohio State University, \\ Columbus, Ohio, USA; ${ }^{2}$ Mount Carmel \\ Medical center, Columbus, Ohio
}

\begin{abstract}
Adjuvant chemotherapy reduces risk of relapse and cancer-related mortality in early stage breast cancer. Over the last decade, taxanes (paclitaxel and docetaxel) have been incorporated into various adjuvant trials and have demonstrated a significant benefit in the management of early stage breast cancer. Clinical trials using combinations of taxanes with targeted therapy have also shown considerable activity in breast cancer. This article reviews the pharmacology of docetaxel, a semi-synthetic taxane, and the clinical trials supporting its use in patients with node-positive breast cancer.
\end{abstract}

Keywords: docetaxel, node-positive breast cancer, post-surgery treatment, taxanes

\section{Introduction}

Nodal involvement is one of the major determinants of the risk of relapse in early stage breast cancer. Adjuvant polychemotherapy improves disease-free and overall survival in early breast cancer. The addition of paclitaxel to standard anthracycline regimen resulted in further improvement in disease-free and overall survival in node-positive patients (Henderson et al 2003) leading to its approval in the management of early stage breast cancer. The key advantage of taxanes is its noninterference with the pharmacokinetics of anthracyclines and its efficacy in anthracyclines-resistant disease.

Docetaxel is an antineoplastic agent belonging to the taxoid family with activity against a wide range of human malignancies (Bridgewater 2004). It is a semi-synthetic agent derived from a European yew tree, Taxus baccata, and was first identified as an alternative to paclitaxel in 1986 (Bissery et al 1995). Its chemical structure differs from paclitaxel with a hydroxyl group replacing the acetyl group at C-10 and variations at the C-13 side chain (Ringel and Horwitz 1991). These structural modifications confer enhanced solubility in aqueous solution, and clinical studies have shown that docetaxel may be active in metastatic cancers resistant to paclitaxel (Valero et al 1998; Michaud et al 2000; Verschraegen et al 2000).

The current recommended dose for docetaxel is $60-100 \mathrm{mg} / \mathrm{m}^{2}$ given as a 1 -hour infusion every 3 weeks. Although initially approved in 1996 for treatment of anthracycline-refractory metastatic breast cancer, docetaxel is now also approved as adjuvant therapy in the management of early, high-risk breast cancer (Ravdin et al 1995; Valero et al 1995). This review will discuss the dosing and toxicity profile of docetaxel and the studies utilizing docetaxel in patients with node-positive breast cancer.

\section{Mechanism of action}

Docetaxel acts by binding to the beta-tubulin subunit of the microtubules, which causes stabilization of tubulin polymerization, resulting in cell cycle arrest at $\mathrm{G} 2 / \mathrm{M}$ phase and inhibition of mitosis (Eisenhauer and Vermorken 1998). Microtubules are the backbone of the cellular skeleton that is essential for the maintenance of cell shape, intracellular transport, reproduction, and neurotransmission. Docetaxel has a high affinity for 
this site, approximately 1.9-fold that of paclitaxel, targeting the centrosome organization and acting on the cell during $\mathrm{S} / \mathrm{G} 2 / \mathrm{M}$ phase. Stabilization of the microtubules disrupts the dynamic reorganization of the microtubules into spindles resulting in formation of abnormal bundles, which inhibit cell proliferation leading to cell death (Rinel and Horwitz 1991; Diaz and Andreu 1993). Compared with paclitaxel, there is a greater uptake of docetaxel into the tumor and slower efflux out of tumor cells, resulting in higher exposure of the tumor cells to docetaxel. This could explain the activity of docetaxel in paclitaxel-resistant tumors.

\section{Toxicity}

Neutropenia is the principal toxicity of docetaxel (Schrijvers et al 1993; Cortes and Pazdur 1995) and has led to dose reduction and use of growth factors in patients with node-positive breast cancer. At a dose of $100 \mathrm{mg} / \mathrm{m}^{2}$, grade 4 neutropenia $\left(<500\right.$ cells $\left./ \mathrm{mm}^{2}\right)$ occurs in $85 \%$ of patients. The most important determinant of neutropenia is the extent of prior therapy. Despite being formulated in polysorbate 80 , hypersensitivity reactions have been reported in approximately $31 \%$ of patients receiving docetaxel without premedications (Schrijvers et al 1993; Cortes and Pazdur 1995). The current recommendation is to give dexamethasone $8 \mathrm{mg}$ twice a day for 3 days, starting a day prior to administration of docetaxel with or without $\mathrm{H}_{1}$ and $\mathrm{H}_{2}$ receptor antagonists given 30 minutes before docetaxel (Piccart et al 1997; Markman 2003). This regimen also reduces the incidence of the unique fluid retention syndrome characterized by edema, weight gain, and third-space fluid collection caused by docetaxel. Evidence indicates that this is due to capillary leak and is usually not apparent until a cumulative dose of $400 \mathrm{mg} / \mathrm{m}^{2}$ is reached. Although fluid retention is reversible, it takes several months to resolve after discontinuation of docetaxel (Piccart et al 1997). Patients developing progressive peripheral edema may be treated with diuretics for symptom relief.

Skin toxicity may occur in as many as 50\%-75\% of patients (Schrijvers et al 1993; Markman 2003). The most common is an erythematous, pruritic, maculopapular rash that affects the forearm, hands, and feet; premedication reduces its incidence. The other less common cutaneous reactions include palmar-plantar erythrodysesthesia and onychodystrophy characterized by brown discoloration, ridging, onycholysis, and, in extreme cases, loss of nail plate. Mild to moderate peripheral neuropathy manifesting as parasthesia, dysesthesia, and pain occurs in approximately $40 \%$ of patients with no prior treatment. Patients with a history of prior cisplatin therapy or alcohol abuse are particularly susceptible (Zimmerman et al 1994, 1995; Piccart et al 1997; Hainsworth et al 1999).

Stomatitis appears to be more frequent with docetaxel than paclitaxel. Hyperlacrimation, although initially classified as an unexpected side effect, is now being increasingly reported. This excessive tearing resulting from cannalicular/nasolacrimal duct stenosis can lead to difficulty reading and driving, and it can affect quality of life (Esmaeli et al 2001). Interventions such as temporary silicone intubation and dacryocystorhinostomy with placement of silicone or pyrex tube may be needed to relieve symptoms (Esmaeli 2005). Severe gastrointestinal side effects are typically rare, although nausea, vomiting, and diarrhea may occur.

\section{Docetaxel in node-positive breast cancer}

The Breast Cancer International Research Group (BCIRG) 001 (Martin et al 2005) adjuvant study randomly allocated 1491 patients with node-positive breast cancer to docetaxel (75 mg/m²), doxorubicin, and cyclophosphamide (TAC) or to fluorouracil, doxorubicin, and cyclophosphamide (FAC) for 6 cycles (Figure 1). At a median follow-up of 55 months, there was a $26 \%$ reduction in the risk of recurrence (hazard ratio $[\mathrm{HR}]=0.74 ; 95 \%$ confidence interval $(\mathrm{CI})=0.60-0.92$; $\mathrm{p}=0.0047)$ in the docetaxel arm. Longer disease-free survival (DFS) was seen in the docetaxel group regardless of the estrogen receptor/progesterone receptor status. This resulted in a benefit in overall survival (OS) for TAC (87\% vs $81 \%$; $\mathrm{p}=0.008$ ) (46) (Table 1). Subgroup analysis showed that patients with fewer than 3 axillary nodes derived a more significant benefit from the addition of docetaxel in terms of DFS (90\% vs 79\%, p = 0.0002) and OS (96\% vs 89\%; $\mathrm{p}=0.006)$. The incidence of grade 3 or 4 neutropenia $(65.5 \%$ vs $49.3 \%, \mathrm{p}<0.001)$ and febrile neutropenia $(24.7 \%$ vs $2.5 \%, \mathrm{p}<0.001)$ was higher in the TAC group. Though grade 3 or 4 infections occurred in $3.9 \%$ of patients treated with TAC and $2.2 \%$ of those treated with FAC $(\mathrm{p}=0.05)$, the rates of sepsis did not differ between the two groups. The investigators concluded that TAC is superior to FAC in node-positive breast cancer patients.

Voegl et al (2004) in a subanalysis of the BCIRG 001 trial investigated the role of growth factor support following neutropenic events. Granulocyte colony stimulating factor (G-CSF) was used as secondary prophylaxis for $87 \%$ (TAC) and $44 \%$ (FAC) patients. The rate of febrile neutropenia per cycle among patients receiving TAC dropped to $3.1 \%$ and FAC to $0.5 \%$ after growth factor support. This retrospective subgroup analysis demonstrated a higher rate of neutropenic 


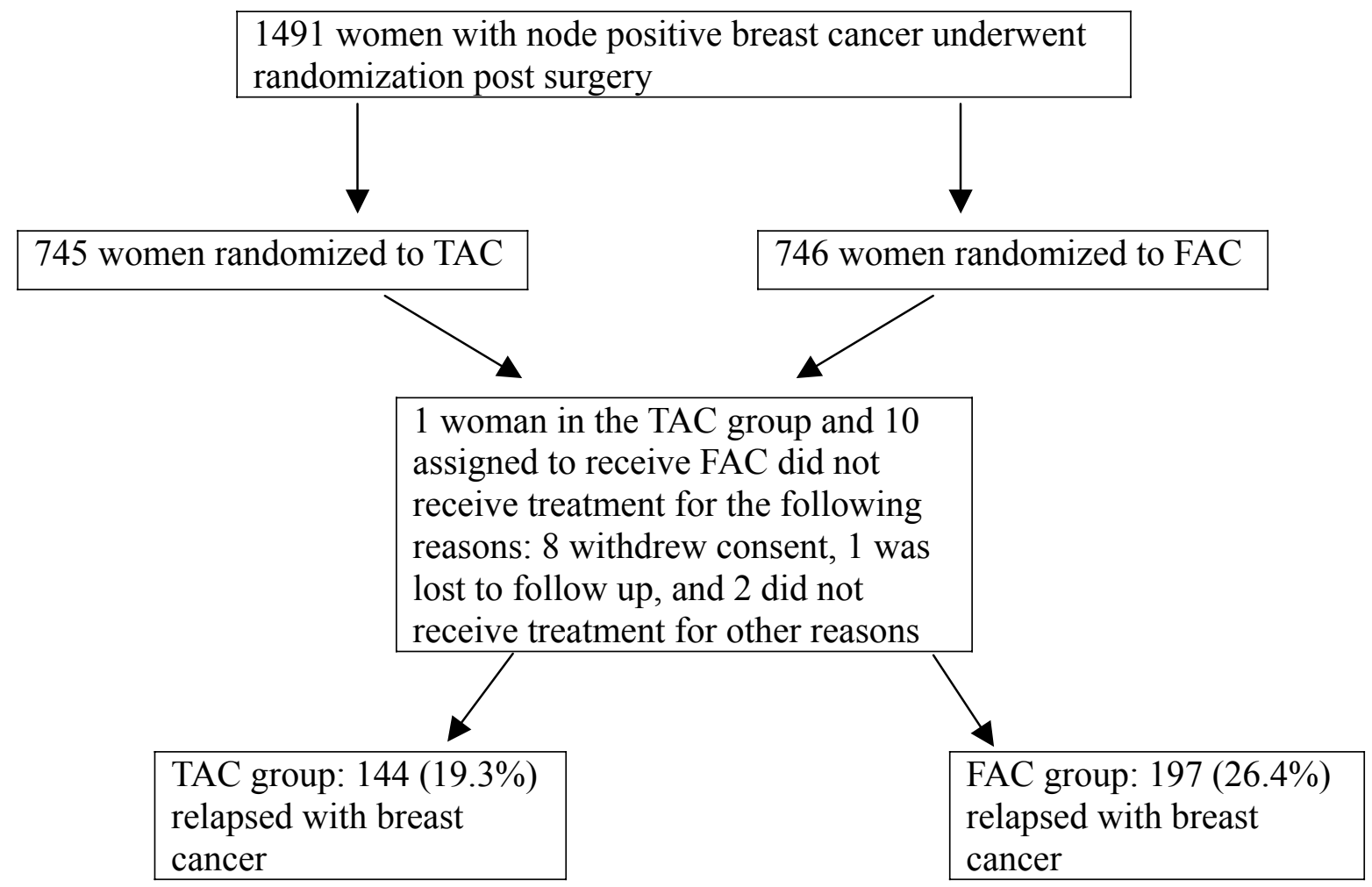

Figure I Adjuvant therapy for node positive breast cancer. Adapted from Martin et al (2005).

Abbreviations: FAC, fluorouracil, doxorubicin, and cyclophosphamide;TAC, docetaxel, doxorubicin, and cyclophosphamide.

fevers in patients on the TAC regimen, and that prophylaxis with G-CSF after the first episode can decrease the incidence of neutropenic complications.

Combination or sequential docetaxel was compared with a nontaxane regimen in 2887 patients with node-positive breast cancer (Crown et al 2006). Patients were randomized to 4 arms: 4 cycles of doxorubicin (A) $\left(75 \mathrm{mg} / \mathrm{m}^{2}\right)$ followed by 3 cycles of cyclophosphamide, methotrexate, and 5 -fluorouracil (CMF), 4 cycles of doxorubicin and cyclophosphamide (AC) followed by 3 cycles of CMF, 3 cycles of doxorubicin followed by 3 cycles of docetaxel (T) (100 $\mathrm{mg} / \mathrm{m}^{2}$ ) followed by 3 cycles of CMF, or, finally, 4 cycles of doxorubicin $\left(50 \mathrm{mg} / \mathrm{m}^{2}\right)$ and docetaxel $\left(75 \mathrm{mg} / \mathrm{m}^{2}\right)$ followed by 3 cycles of CMF. The primary end-point of the study was DFS. The $\mathrm{A} \rightarrow \mathrm{T} \rightarrow \mathrm{CMF}$ arm demonstrated a significantly improved DFS over the AT $\rightarrow \mathrm{CMF}(\mathrm{p}=0.047)$ and $\mathrm{A} \rightarrow \mathrm{CMF}(\mathrm{p}=0.035)$ arms.

The French FNCLCC-PACS 01 trial randomly assigned 1999 patients with node-positive breast cancer to 6 cycles of fluorouracil $500 \mathrm{mg} / \mathrm{m}^{2}$, epirubicin $100 \mathrm{mg} / \mathrm{m}^{2}$, and cyclophosphamide $500 \mathrm{mg} / \mathrm{m}^{2}$ (FEC) or to 3 cycles of the same regimen followed by 3 cycles of $100 \mathrm{mg} / \mathrm{m}^{2}$ of docetaxel (D) (Roché et al 2006). After a median follow-up of 60 months, switching over to docetaxel after 3 cycles of FEC resulted in an improvement in 5-year disease-free $(78.4 \%$ vs $73.2 \% ; \mathrm{p}=0.01)$ and overall survival $(90.7 \%$ vs $86.7 \% ; \mathrm{p}=0.02)$. The rate of distant metastasis was lower in patients who received FEC-D (18.1\% vs $14.9 \%)$. Similar to the BCIRG study, among the patients with 1-3 positive nodes there was a significant reduction in the

Table I Phase III trials of docetaxel as adjuvant therapy in patients with node-positive breast cancer

\begin{tabular}{lllll}
\hline Study & Regimen & Number of patients & Disease-free survival & Overall survival \\
\hline BCIRG 00I & TAC vs FAC & $\mathrm{I}, 49 \mathrm{I}$ & $75 \%$ vs $68 \% \mathrm{p}=0.00 \mathrm{I}$ & $87 \%$ vs $81 \% \mathrm{P}=0.008$ \\
FNCLCC PACS 0I & FEC $\times 3 \rightarrow$ docetaxel $\times 3$ vs FEC X 6 & $\mathrm{I}, 999$ & $78 \%$ vs $74 \% \mathrm{p}=0.04 \mathrm{I}$ & $91 \%$ vs $87 \% \mathrm{p}=0.05$ \\
BIG 2-098 & $\mathrm{A} \rightarrow \mathrm{T}+$ AT vs A + AC & 2,887 & HR $0.86 \mathrm{p}=0.05 \mathrm{I}$ & $\mathrm{HR} 0.92 \mathrm{p}=0.34$ \\
\hline
\end{tabular}

Abbreviations: HR, hazard ratio; A, doxorubicin; AC, doxorubicin and cyclophosphamide; AT, adriamycin, docetaxel; FAC, fluorouracil, doxorubicin, and cyclophosphamide; TAC, docetaxel, doxorubicin, and cyclophosphamide; FEC, fluorouracil, epirubicin, and cyclophosphamide. 
risk of relapse with $\mathrm{FEC} \rightarrow \mathrm{D}$ compared with $\mathrm{FEC}$ alone $(p=0.04)$. Similar benefits were not observed in women with more than 3 positive lymph nodes. Interestingly, the benefit in DFS was more apparent in women age 50 years or older $(p=0.001)$ than in younger women $(p=0.65)$. The addition of docetaxel was associated with a higher rate of febrile neutropenia, but the overall incidence of grade 3 or 4 neutropenia in cycles 4-6 was higher in the FEC group (20.2\% vs $10.9 \%$ ) The use of growth factors was significantly increased in patients receiving 6 cycles of FEC ( $p<0.001)$ Treatment with FEC-D was associated with significantly fewer cardiac events.

Safety data from ongoing studies have been reported. The data on efficacy are awaited. The BCIRG 005 study randomized 3298 patients with node-positive breast cancer to either 6 cycles of docetaxel $\left(75 \mathrm{~g} / \mathrm{m}^{2}\right)$, doxorubicin, and cyclophosphamide (TAC) or 4 cycles of doxorubicin and cyclophosphamide, followed by docetaxel (T) $\left(100 \mathrm{mg} / \mathrm{m}^{2}\right)$. Both regimes were administered every 3 weeks (Eiermann et al 2005). Patients were given prophylactic ciprofloxacin and G-CSF was administered at the time of febrile neutropenia. There was an increased incidence of febrile neutropenia in the TAC group (17.9\% vs $8.5 \%)$, and more neurotoxicities and myalgias in the AC followed by $\mathrm{T}$ arm.

In the phase III PACS 04 trial, patients were randomized to receive 6 cycles of FEC 100 or 6 cycles of epirubicin and docetaxel $\left(75 \mathrm{mg} / \mathrm{m}^{2}\right)(\mathrm{ET})$. The patients in the ET arm had an increased incidence of febrile neutropenia (31\% vs $10.7 \%$ ) while there was a significant decline in the left ventricular ejection fraction seen in the FEC arm (Spielmann et al 2006).

A phase II trial (Piedbois et al 2005) evaluated grade 4 toxicities of 6 cycles of docetaxel $\left(75 \mathrm{mg} / \mathrm{m}^{2}\right)$, epirubicin $\left(75 \mathrm{mg} / \mathrm{m}^{2}\right)$, and cyclophosphamide $\left(500 \mathrm{mg} / \mathrm{m}^{2}\right)$ every 3 weeks (control group) or 4 cycles of epirubicin $\left(100 \mathrm{mg} / \mathrm{m}^{2}\right)$ and cyclophosphamide $\left(600 \mathrm{mg} / \mathrm{m}^{2}\right)$ followed by 4 cycles of docetaxel $\left(100 \mathrm{mg} / \mathrm{m}^{2}\right)$ every 2 weeks (arm A) or 4 cycles of docetaxel followed by 4 cycles of epirubicin and cyclophosphamide (arm B), in 100 patients with node-positive breast cancer. Prophylactic pegfilgastrim was administered. Sixty-one patients were evaluated for toxicity. Grade 3/4 toxicities of the control arm compared with arm A or arm B included neutropenia ( $38 \%$ vs $35 \%$ vs 33\%), hand foot syndrome ( $0 \%$ vs $25 \%$ vs $33 \%$ ), neurotoxicity ( $0 \%$ vs $15 \%$ vs $6 \%$ ), and nausea ( $0 \%$ vs 155 vs $6 \%$ ) respectively. The incidence of febrile neutropenia was highest in the control group ( $14 \%$ vs $5 \%$ vs $0 \%$ ). These regimens remain to be tested in phase III trials.
An ongoing Italian study randomized patients to receive either 4 cycles of EC (epirubicin $120 \mathrm{mg} / \mathrm{m}^{2}$ and cyclophosphamide $600 \mathrm{mg} / \mathrm{m}^{2}$ ) Q21 days or 4 cycles of docetaxel $\left(100 \mathrm{mg} / \mathrm{m}^{2}\right)$ followed by 4 cycles of EC. Initial data on toxicity revealed a higher rate of grade 4 neutropenia and febrile neutropenia in the docetaxel arm with an increased incidence of grade 3/4 nausea/vomiting in the EC arm (Valeria et al 2001).

In summary, studies in node-positive breast cancer with docetaxel given concurrently or sequentially with anthracyclines improved disease-free survival. This benefit was most evident in patients with fewer than 3 positive nodes. Prophylactic use of growth factors should be considered in patients receiving docetaxel in combination with other myelosuppressive chemotherapy.

\section{Docetaxel in studies including both node-negative and node-positive breast cancer}

A number of important studies addressing docetaxel in the adjuvant setting have included both node-negative and nodepositive patients. The Eastern Cooperative Oncology Group (ECOG) trial E2197 randomized 2952 women to 4 cycles of AT (adriamycin $60 \mathrm{mg} / \mathrm{m}^{2}$, docetaxel $60 \mathrm{mg} / \mathrm{m}^{2}$ ) or 4 cycles of AC (adriamycin $60 \mathrm{mg} / \mathrm{m}^{2}$, cyclophosphamide $600 \mathrm{mg} / \mathrm{m}^{2}$ ), administered every 3 weeks for 4 cycles. Thirty-five percent of the patients were lymph node-positive. After a median follow-up of 53 months, DFS was identical (87\%) in both groups of patients. The incidence of febrile neutropenia was higher with AT (19\%) than with AC (6\%). There were 3 treatment related-deaths in the AT arm. The difference in efficacy could be attributed to the lower dose of docetaxel and fewer node-positive patients in this ECOG trial.

Jones et al (2006) randomized 1601 patients with stage I to III operable invasive breast cancer to 4 cycles of AC $\left(60 \mathrm{mg} / \mathrm{m}^{2}\right.$ and $600 \mathrm{mg} / \mathrm{m}^{2}$ respectively) or TC $\left(75 \mathrm{mg} / \mathrm{m}^{2}\right.$ and $600 \mathrm{mg} / \mathrm{m}^{2}$ respectively), administered every 3 weeks in the adjuvant setting. The 5-year DFS rate was superior for $\mathrm{TC}$ than $\mathrm{AC}(86 \%$ vs $80 \%, \mathrm{p}=0.15)$. The patients in the $\mathrm{AC}$ arm experienced more grade 1 to 4 nausea and vomiting $(\mathrm{p}<0.01)$, whereas more fever and neutropenia was observed with TC (5\%) vs AC $(2.5 \%, \mathrm{p}=0.07)$. In the AC group, 1 patient died form congestive heart failure and 1 patient in the TC group died of sepsis and neutropenia. In a subgroup analysis, both node-negative $(\mathrm{TC}=239, \mathrm{AC}=248)$ and node-positive ( $\mathrm{TC}=267, \mathrm{AC}=262$ ) patients seemed to favor the TC arm. The HR for node-negative patients was 0.73 and for the node-positive patients was 0.67 , but this 
difference was statistically significant only in patients with node-positive disease $(\mathrm{p}<0.05)$. The study was not powered to detect differences in subgroups.

The North American Breast Cancer Intergroup Trial E1199 compared the effectiveness of adjuvant paclitaxel with docetaxel, and the effectiveness of 3-weekly with weekly adjuvant taxane therapy in 4988 patients with operable breast cancer. Patients received 4 cycles of AC $\left(60 \mathrm{mg} / \mathrm{m}^{2}\right.$ and $600 \mathrm{mg} / \mathrm{m}^{2}$ respectively) every 3 weeks, followed by either: paclitaxel $175 \mathrm{mg} / \mathrm{m}^{2}$ every 3 weeks $\times 4$, paclitaxel $80 \mathrm{mg} / \mathrm{m}^{2}$ weekly $\times 12$, docetaxel $100 \mathrm{mg} / \mathrm{m}^{2}$ every 3 weeks $\times 4$, or docetaxel $35 \mathrm{mg} / \mathrm{m}^{2}$ weekly $\times 12$. Most patients $(88 \%)$ had lymph node-positive disease. After a median follow-up of 46.5 months, there were no significant differences in HR comparing taxane $(0.985 ; \mathrm{p}=0.83)$ or schedule $(1.043$; $\mathrm{p}=0.54$ ) for DFS. In an exploratory analysis, weekly paclitaxel was found to have superior DFS compared with 3 -weekly paclitaxel (HR 1.20, p < 0.06). The docetaxel arms did not differ significantly from 3-weekly paclitaxel but had more Grade III and IV hematological toxicities.

\section{Combinations with trastuzumab}

Trastuzumab is a humanized monoclonal antibody against the HER2 protein, which is overexpressed in $15 \%-25 \%$ of breast carcinomas. The role of trastuzumab is established in metastatic breast cancer where it improves survival when administered with chemotherapy (Slamon et al 2001). This benefit extended to the adjuvant setting with 2 large phase III randomized placebo-controlled trials demonstrating a significant improvement in DFS with the addition of trastuzumab to conventional chemotherapy (Piccart-Gebhart et al 2005; Romond et al 2005).

Interestingly, data obtained from in vitro experiments show that docetaxel exhibits synergistic effects against breast cancer cells when administered with trastuzumab, in contrast to the additive effects it exhibits when given with paclitaxel.

In early trials of trastuzumab with chemotherapy, $27 \%$ of patients treated concurrently with trastuzumab and anthracyclines, 13\% with trastuzumab and paclitaxel, and 5\% with trastuzumab alone had cardiotoxic events (Slamon et al 2001). To overcome the risk of cardiotoxicity, the BCIRG 006 trial randomized patients into 3 arms: comparing doxorubicin and cyclophosphamide followed by docetaxel $(\mathrm{AC} \rightarrow \mathrm{T})$ with doxorubicin and cyclophosphamide followed by docetaxel and trastuzumab $(\mathrm{AC} \rightarrow \mathrm{TH})$ with docetaxel, carboplatin, and trastuzumab $(\mathrm{TCH})$. The combination of docetaxel and trastuzumab was well tolerated. After a median follow-up of
36 months, both $\mathrm{AC} \rightarrow \mathrm{TH}$ and $\mathrm{TCH}$ significantly improved the DFS and OS over the control (Slamon et al 2005).

A prospectively designed subgroup analysis of the BCIRG 001 trial showed that Her 2-neu positive patients treated with TAC had a significant reduction in risk $(0.60$, CI 0.41-0.88) compared with FAC (Trudeau et al 2005). To date, there is insufficient evidence to conclude that HER 2-neu positive breast cancer patients derive superior benefit from this combination and it still remains to be tested in well-designed randomized clinical trials.

\section{Conclusions}

Adjuvant trials with docetaxel have demonstrated a significant reduction in recurrence and longer disease-free interval in patients with node-positive breast cancer (Martin et al 2005). The survival benefit was more apparent in patients with fewer than 3 axillary lymph nodes and in women older than 50 years of age (Martin et al 2005; Roché et al 2006). The most common side effect is myelosuppression and neutropenic fevers. The incidence of this can be reduced by prophylaxis with growth factors. Hypersensitive reactions and fluid retention are minimized by administration of dexamethasone and histamine receptor antagonists.

Addition of trastuzumab to docetaxel appears promising. Combination of docetaxel with antiangiogeneic agents and trastuzumab is currently under investigation. Further studies are needed to define the most effective and safe dosing regimen of docetaxel to maximize benefit and reduce the incidence of neutropenic complications in patients with node-positive breast cancer.

\section{References}

Bissery MC, Nohynek G, Sanderink GJ, et al. 1995. Docetaxel (Taxotere): a review of preclinical and clinical experience. Part I: Preclinical experience. Anticancer Drugs, 6:339-55, 363-8.

Bridgewater. 2004. Taxotere prescribing information. Aventis Pharmaceuticals Inc. NJ.

Cortes JE and Pazdur R. 1995. Docetaxel. J Clin Oncol, 13:2643-55.

Crown JP, Francis P, Di Leo A, et al. 2006. Docetaxel (T) given concurrently with or sequentially to anthracycline-based (A) adjuvant therapy (adjRx) for patients (pts) with node-positive $(\mathrm{N}+$ )breast cancer $(\mathrm{BrCa})$, in comparison with non-T adjRx: First results of the BIG 2-98 Trial at 5 years median follow-up (MFU). J Clin Oncol, 24(18S):Abstract 519.

Diaz JF and Andreu JM. 1993. Assembly of purified GDP-tubulin into microtubules induced by taxol and taxotere: reversibility, ligand stoichiometry, and competition. Biochemistry, 32:2747-55.

Eiermann W, Pienkowski T, Crown J, et al. 2005. Phase 111 randomized trial comparing docetaxel in combination with doxorubicin and cyclophosphamide versus doxorubicin and cyclophosphamide followed by docetaxel in Her-2/neu negative early breast cancer patients with positive axillary lymph nodes. Proc SABCS: Abstract 1069.

Eisenhauer EA, Vermorken JB. 1998. The taxoids. Comparative clinical pharmacology and therapeutic potential. Drugs, 55:5-30. 
Esmaeli B, Valero V, Ahmadi MA, et al. 2001. Canalicular stenosis secondary to docetaxel (taxotere): a newly recognized side effect. Ophthalmology, 108:994-5.

Esmaeli B. 2005. Management of excessive tearing as a side effect of docetaxel. Clin Breast Cancer, 5:455-7.

Hainsworth JD, Burris HA 3rd, Greco FA. 1999. Weekly administration of docetaxel (Taxotere): summary of clinical data. Semin Oncol, 26(Suppl 10):19-24.

Henderson IC, Berry DA, Demetri GD, et al. 2003. Improved outcomes from adding sequential Paclitaxel but not from escalating Doxorubicin dose in an adjuvant chemotherapy regimen for patients with node-positive primary breast cancer. J Clin Oncol, 21:976-83.

Jones SE, Savin MA, Holmes FA, et al. 2006. Phase III trial comparing doxorubicin plus cyclophosphamide with docetaxel plus cyclophosphamide as adjuvant therapy for operable breast cancer. J Clin Oncol, 24:5381-7.

Markman M. Managing taxane toxicities. 2003. Support Care Cancer, 11:144-7.

Martin M, Pienkowski T, Mackey J, et al. 2005. Adjuvant docetaxel for node-positive breast cancer. $N$ Engl J Med, 352:2302-13.

Michaud LB, Valero V, Hortobagyi G. 2000. Risks and benefits of taxanes in breast and ovarian cancer. Drug Safety, 23:401-28.

Piccart MJ, Klijn J, Paridaens R, et al. 1997. Corticosteroids significantly delay the onset of docetaxel-induced fluid retention: final results of a randomized study of the European Organization for Research and Treatment of Cancer Investigational Drug Branch for Breast Cancer. J Clin Oncol, 15:3149-55.

Piccart-Gebhart MJ, Procter M, Leyland-Jones B, et al. 2005. Trastuzumab after adjuvant chemotherapy in HER2-positive breast cancer. $N$ Engl $J$ Med, 353:1659-72.

Piedbois P-P, Serin D, Priou F, et al. 2005. AERO-B03: A randomized phase 11 trial of dose-dense, docetaxel in node-positive breast cancer. $J$ Clin Oncol, 23(16S):Abstract 647.

Ravdin PM, Burris HA 3rd, Cook G, et al. 1995. Phase II trial of docetaxel in advanced anthracycline-resistant or anthracenedione-resistant breast cancer. J Clin Oncol, 13:2879-85.

Ringel I, Horwitz SB. 1991. Studies with RP 56976 (taxotere): a semisynthetic analogue of taxol. J Natl Cancer Inst, 83:288-91.

Roché H, Fumoleau P, Spielmann M, et al. 2006. Sequential adjuvant epirubicin-based and docetaxel chemotherapy for node-positive breast cancer patients: the FNCLCC PACS 01 Trial. J Clin Oncol, 24:5664-71.

Romond EH, Perez EA, Bryant J, et al. 2005. Trastuzumab plus adjuvant chemotherapy for operable HER2-positive breast cancer. $N$ Engl J Med, 353:1673-84.
Schrijvers D, Wanders J, Dirix L, et al. 1993. Coping with toxicities of docetaxel (Taxotere). Ann Oncol, 4:610-11.

Slamon D, Eiermann W, Robert N, et al. 2005. Phase III randomized trial comparing doxorubicin and cyclophosphamide followed by docetaxel $(\mathrm{AC} \Rightarrow \mathrm{T})$ with doxorubicin and cyclophosphamide followed by docetaxel and trastuzumab $(\mathrm{AC} \Rightarrow \mathrm{TH})$ with docetaxel, carboplatin and trastuzumab (TCH) in HER2 positive early breast cancer patients: BCIRG 006 study. Proc SABCS:Abstract 1.

Slamon DJ, Leyland-Jones B, Shak S, et al. 2001. Use of chemotherapy plus a monoclonal antibody against HER2 for metastatic breast cancer that overexpresses HER2. N Engl J Med, 344:783-92.

Spielmann M, Roché H, Delozier T, et al. 2006. Safety analysis from PACS 04-A phase 111 trial comparing 6 cycles of FEC 100 with 6 cycles of ET75 for node-positive early breast cancer patients followed by sequential trastuzumab in HER2 patients: Preliminary results. $J$ Clin Oncol, 24(18S):Abstract 632.

Trudeau M, Charbonneau F, Gelmon K, et al. 2005. Selection of adjuvant chemotherapy for treatment of node-positive breast cancer. Lancet Oncol, 6:886-98.

Valeria F, Ester E, Modesto D'Aprile, et al. 2001. A feasibilty study of a sequential dose-dense adjuvant regimen with epidoxorubicin followed by docetaxel followed by high-dose cyclophosphamide for early breast cancer with 4 or more lymph node metastases. Annals of Oncol, 12(4S):52.

Valero V, Holmes FA, Walters RS, et al. 1995. Phase II trial of docetaxel: a new, highly effective antineoplastic agent in the management of patients with anthracycline-resistant metastatic breast cancer. J Clin Oncol, 13:2886-94.

Valero V, Jones SE, Von Hoff DD, et al. 1998. A phase II study of docetaxel in patients with paclitaxel-resistant metastatic breast cancer. $J$ Clin Oncol, 16:3362-8.

Verschraegen CF, Sittisomwong T, Kudelka AP, et al. 2000. Docetaxel for patients with paclitaxel-resistant Mullerian carcinoma. J Clin Oncol, 18:2733-9.

Vogel CL, Mackey JR, Martin M, et al. 2004. The role of growth factor support following neutropenic events $n$ early stage breast cancer (BC) patients treated with adjuvant docetaxel, doxorubicin, and cyclophosphamide (TAC): A sub-analysis of BCIRG 001. J Clin Oncol, 22(14S): Abstract 677.

Zimmerman GC, Keeling JH, Burris HA, et al. 1995. Acute cutaneous reactions to docetaxel, a new chemotherapeutic agent. Arch Dermatol, 131:202-6.

Zimmerman GC, Keeling JH, Lowry M, et al. 1994. Prevention of docetaxelinduced erythrodysesthesia with local hypothermia. $J$ Natl Cancer Inst, 86:557-8. 